Dialectologia 14 (2014), 97-114.

ISSN: 2013-2247

Received 28 September 2013.

Accepted 18 December 2013.

\title{
EL NARCOLENGUAJE EN EL HABLA ACTUAL DE BAJA CALIFORNIA, MÉXICO
}

\author{
Rafael Saldívar ArReola ${ }^{1}$ \& Ignacio Rodríguez SánChez ${ }^{2}$ \\ Universidad Autónoma de Baja California ${ }^{1}$ / Universidad Autónoma de Querétaro ${ }^{2}$ \\ rafaelsaldivar@uabc.edu.mx / igrodsan@uaq.mx
}

Resumen

La influencia del tráfico de drogas ha sido evidente en el habla cotidiana en México. El presente estudio cuantitativo se enfoca en la dinámica que permite que el léxico del narco se traslade de la marginalidad al habla común, en especial en la Ciudad de Mexicali, ciudad fronteriza con el Estado de California.

Con base en un corpus de textos escritos y orales, que incluyen prensa local, blogs, "narcocorridos" y entrevistas con personas involucradas en el tráfico y consumo de drogas, del cual se obtuvieron más de quinientos términos referentes al narcotráfico, se hizo una selección de sesenta términos para establecer el nivel de reconocimiento de este tipo de léxico en una muestra estratificada de habitantes de Mexicali. Este análisis constituye una mirada a algunos de los factores causales en la compleja dinámica de diseminación del léxico del narco en la sociedad.

\section{Palabras clave}

variación léxica, corpus, análisis cuantitativo, narcoléxico

\section{DRUG-RELATED LANGUAGE IN CURRENT SPEECH IN BAJA CALIFORNIA, MEXICO}

\section{Abstract}

The influence of drug trafficking has been evident in Mexico's common speech. This study analyzes the dynamics that enable the terms and expressions of the drugs world to transfer from marginality to mainstream speech in the city of Mexicali, in the Mexican border with California. 
The work is based on a corpus formed by texts of local press, essays on drug trafficking, local literary texts, blogs, and "narcocorridos" (songs about drug kingpins) - and by interviews with people involved in the commerce and use of drugs. Out of more than five hundred terms obtained in the corpus, sixty terms were selected according to their frequency (high, low and specialized) to establish the level of awareness of this language on a stratified sample of people in Mexicali. This analysis constitutes a glimpse of some causal factors in the complex dynamics of dissemination of the drug-related language in the society of Mexicali.

\section{Keywords}

drug-related language, lexical variation, corpus, quantitative analysis

\section{Introducción}

Uno de los fenómenos sociales que ha venido a trastocar la cotidianidad en México es la operación de los grupos dedicados a la producción y al transporte de drogas ilegales, así como las acciones punitivas del gobierno hacia estos grupos.

Esta presencia palpable del fenómeno narco en la región se manifiesta también en la transformación del lenguaje, y este a su vez colabora en la representación social de la realidad mexicana actual; en especial de la zona fronteriza.

Palabras como "pozolear", "enteipado", "encajuelados" y "levantones" hablan de una realidad nueva (Roldán Quiñoñez 2006), generando un imaginario social caracterizado por la violencia y la descomposición social. El narcolenguaje, como reflejo del fenómeno narco, comparte la aparente informidad y crudeza que causa perplejidad entre quienes atestiguan su desarrollo. Como afirma Baker (2010), "el pánico moral se caracteriza por la identificación de un problema que se percibe como una amenaza para una comunidad o una sección de los valores o intereses de una comunidad".

Este tipo de lenguaje se puede clasificar como argot, en tanto es un "cuerpo de vocabulario no estándar utilizado por algunos grupos sociales aislados, generalmente en oposición a las autoridades, que es potencialmente obscuro para quien solamente conoce la variedad del lenguaje dominante" (Blake 2010). Lara (2006) utiliza la palabra caló para referirse a este tipo de lenguaje, caracterizándolo como vocabulario de los delincuentes "en especial ladrones o rateros, comerciantes de drogas; no los grandes narcotraficantes, que suelen ser políticos o financieros respetados". No obstante, en México este tipo de 
lenguaje parece romper las barreras sociales e irse adaptando al habla común, por una parte a través de los medios de comunicación, pero también emergiendo en distintos ámbitos sociales en los que la presencia no solamente de traficantes, sino también de un cada vez mayor número de consumidores de drogas, hace necesario el tener un código a través del cual conceptualizar esta nueva realidad. Por ello, se optó por no utilizar ni el término argot ni el término caló para referirse al objeto de estudio de este trabajo y simplemente se usará narcolenguaje.

A continuación en la Tabla (1) en la que se pueden ver las diferentes fuentes y esferas de uso del narcolenguaje y algunas de las características de cada una de ellas.

\begin{tabular}{|l|l|l|}
\hline Fuentes & Informantes & Características del lenguaje \\
\hline $\begin{array}{l}\text { Prensa, ensayo investigativo, } \\
\text { discursos políticos }\end{array}$ & $\begin{array}{l}\text { Periodistas, investigadores, } \\
\text { autoridades. }\end{array}$ & $\begin{array}{l}\text { Lenguaje muy difundido, expresiones del discurso } \\
\text { oficial, demuestra distancia con el narco. }\end{array}$ \\
\hline Blogs, Narcocorridos & $\begin{array}{l}\text { Traficantes, personas con } \\
\text { afinidad a la narcocultura }\end{array}$ & $\begin{array}{l}\text { Lenguaje muy coloquial, medianamente difundido, } \\
\text { demuestra afinidad con el narco. }\end{array}$ \\
\hline Entrevistas & $\begin{array}{l}\text { Policías, consumidores, } \\
\text { traficantes }\end{array}$ & $\begin{array}{l}\text { Lenguaje críptico, poco difundido, demuestra una } \\
\text { relación directa con el narco. }\end{array}$ \\
\hline
\end{tabular}

Tabla 1. Fuentes y esferas de uso del narcolenguaje.

\section{Metodología}

Para llevar a cabo este trabajo fue necesario primero conformar un corpus especializado, posteriormente un análisis lexicológico de los términos encontrados y finalmente un estudio sociolingüístico que pretende revisar cómo el narcolenguaje se inserta en la sociedad a través de diversos mecanismos, y cómo diferentes variables sociales se relacionan con el conocimiento del narcolenguaje en la sociedad de Mexicali.

\subsection{Conformación de corpus}

La primera etapa del trabajo consistió en la compilación de los textos obtenidos de publicaciones locales impresas y electrónicas. Los diarios consultados son: La Crónica y La Voz de la Frontera, ambos de Mexicali; Frontera y El Sol, de Tijuana; los semanarios Zeta, de Tijuana, y El Pionero, del Valle de Mexicali; y los noticiarios electrónicos 
Radiopatrulla.com y AFNtijuana.info, de Mexicali y Tijuana, respectivamente. El número de textos fue de aproximadamente diez números de cada publicación, incluyendo todas las secciones de estas.

Más adelante, se integraron algunos libros de autores bajacalifornianos de ficción, que tratan el tema de la delincuencia. Posteriormente se resolvió incluir textos de no ficción y que analizaran el fenómeno del narco. Se incluyeron primeramente textos de escritores que estuvieran vinculados directamente con el estado de Baja California; también se incorporaron libros de autores principalmente del estado de Sinaloa. En total se incorporaron diez libros especializados en el lenguaje narco.

Hasta ese punto se habían agregado textos que, si bien versaban sobre el tema del narcotráfico, lo hacían desde una perspectiva externa. Entonces surgió la necesidad de incluir muestras de lenguaje que mostraran la subjetividad de quienes forman parte activa de este fenómeno. La primera elección en este sentido fue incluir los comentarios vertidos en algunos blogs (AFNtijuana.info, narcotijuana.com), los cuales aparentemente son utilizados por miembros de bandas delictivas para expresarse.

Baker (2010) afirma que el lenguaje del internet no es representativo del habla oral o escrito, sino que es una variedad (o conjunto de variedades) en sí mismo. De hecho los términos y expresiones encontrados en estas fuentes manifestaron características mucho más cercanas a la lengua oral que a la escrita. Por esta razón, en el estudio sociolingüístico que se realizó posteriormente, se decidió fusionar estas dos categorías en una sola.

Por último, en esta necesidad de encontrar textos que ofrecieran muestras de lenguaje auténticamente utilizado por miembros de la cultura del narco, se pensó en incluir un subcorpus de narcocorridos. El lenguaje utilizado en los corridos es la muestra más pura del narcolenguaje, ya que son canciones de ellos y para ellos. A través de los narcocorridos se destila la esencia de la narcocultura. De esta forma se llevó a cabo una recopilación de narcocorridos de los artistas más populares en ese medio para tener una referencia bastante confiable de términos pertenecientes a la narcocultura.

La compilación del corpus especializado se dio por concluida con un número de 2.264.796 muestras y 90.311 tipos. 


\section{- Entrevistas}

Una vez que se terminó el proceso de recopilación de términos que se asumían como pertenecientes al narcolenguaje, fue necesario constatar esta pertenencia. Se establecieron entrevistas con personas que, de alguna forma, son parte de la comunidad del narco; bien por ser traficantes, consumidores, policías o investigadores del fenómeno.

Se entrevistaron a seis personas.

La primera persona es una mujer que se desempeña como investigadora de tiempo completo en la Universidad Autónoma de Baja California y su principal objeto de estudio es el fenómeno del narcotráfico.

Después se entrevistó a un hombre (alrededor de cuarenta años) que trabaja como comerciante de drogas independiente.

La tercera persona es un joven de alrededor de 25 años, ciudadano americano. Él trabaja para una célula instalada en la ciudad de Mexicali y su trabajo consiste en recoger cargas de droga que se lanzan en la zona desértica del Valle Imperial, en el lado estadounidense de la frontera con Mexicali.

Otra persona que se entrevistó también es un joven de aproximadamente 25 años. El trabajo de esta persona es recolectar dinero en la zona de Los Ángeles California y entregarlo a un grupo que opera en la ciudad de Mexicali.

También se entrevistó a un comandante de la Policía Ministerial del Estado, de aproximadamente cincuenta años, adscrito a la ciudad de Mexicali.

Finalmente se entrevistó a un joven de 23 años quien ha sido consumidor de drogas por algunos años, y se ha involucrado de manera indirecta con personas inmiscuidas en el tráfico de drogas.

Las entrevistas consistían de dos partes. En la primera, se les pedía a los informantes que señalaran si los términos que se habían identificado como narcolenguaje mediante el corpus en realidad las conocían y utilizaban. Si por lo menos dos de los entrevistados consideraban que los términos y expresiones en realidad se utilizaban, éstos se registraban como términos aceptados, los que no se descartaban.

La segunda parte de la entrevista, cuyo objetivo era el de recabar términos que se usaban en la narcocultura y que se hubiesen pasado por alto en el proceso de 
recopilación inicial, se apegaba al formato de una entrevista sociolingüística. Las entrevistas fueron guiadas en razón de ciertos temas centrales que se habían identificado en los análisis previos; por ejemplo drogas, mecanismos de operación de los cárteles, armas, etc. De esta forma, se pudieron obtener términos de ámbitos de especialización diversos; desde expresiones utilizadas por quienes se dedican al comercio de sustancias ilegales en su operación cotidiana, hasta expresiones usadas por los delincuentes que se encuentran detenidos en las prisiones locales.

\subsection{Cuestionario sociolingüístico}

Esta prueba se conformó de dos partes, una con los ítems léxicos que servirían para la medición del narcolenguaje, y otra con variables sociolingüísticas. A continuación se detallan las dos partes del instrumento de medición.

\subsection{1 Ítems lingüísticos}

Uno de los factores que se consideraron para la selección de los sesenta ítems fue el poder establecer alguna diferencia entre los ítems pertenecientes al registro oral, y aquellos pertenecientes al registro escrito. Esta diferencia al mismo tiempo permitió acotar otro rasgo importante en elementos léxicos analizados, la frecuencia. Esto se determinó con el supuesto de que la frecuencia dentro del corpus debería reflejar también la frecuencia de uso en el habla de la población de Mexicali.

De tal forma, se obtuvieron tres categorías:

- Los términos de una frecuencia mayor a 100 en el corpus escrito se descartaron, ya que se asumió que serían palabras altamente difundidas, algunas de las cuales incluso han sido ya incluidas en el Diccionario de la Real Academia de la Lengua. Por ejemplo "cuerno de chivo", o "narcocorrido" (Real Academia de la Lengua Española 2010).

- Los términos de una frecuencia entre 99 y uno, son 61 valores diferentes, la mediana de esos valores es 35. De esta forma, se obtuvieron dos grupos de frecuencia: alta, para palabras de una frecuencia mayor a 35 y baja, para palabras con una frecuencia 
menor. El grupo de palabras de alta frecuencia es de 17 elementos y el de baja frecuencia es de 23.

- En cuanto a las expresiones de registro oral, obtenidos a través de las entrevistas, se consideraron únicamente aquellas que fueron utilizadas por más de tres informantes. Este grupo quedó conformado por 20 términos.

Lo que se buscaba evaluar con este instrumento era el conocimiento de los participantes de los términos del narcolenguaje incluidos. Se les pidió a los entrevistados que dijeran el significado de los ítems incluidos. Se consideraron tres posibles puntuaciones para determinar el nivel de conocimiento de las palabras incluidas: dos puntos cuando conocían las palabras, un punto cuando mostraban un conocimiento parcial, y cero cuando no las conocían.

\subsubsection{Variables socio-culturales}

Además de los ítems léxicos, se incluyeron en el cuestionario algunos reactivos que miden ciertas variables sociales que consideramos permiten vislumbrar algunos factores causales de las dinámicas involucradas en el reconocimiento de dicha terminología. Las variables son las siguientes: a) género, b) edad, c) estatus socioeconómico, d) la cercanía de las personas con el fenómeno narco, y e) hábitos de consumo de algunas manifestaciones de la narcocultura. De acuerdo a Geeraerts (2008) el conocimiento semántico se distribuye de una manera no homogénea entre los miembros de una comunidad de habla.

La prueba se aplicó a 83 personas en Mexicali; 47 hombres y 36 mujeres, de edades comprendidas entre los 15 y 68 años. Las edades tuvieron una media de 30.2 y una desviación típica de 13.31. Se incluyeron individuos de tres estratos sociales; 28 personas de nivel socioeconómico bajo, 30 de nivel medio y 25 de nivel alto. 


\section{Análisis de los datos}

El puntaje máximo posible de la muestra era de 120. La media que se obtuvo fue de 60.42 y la mediana de 60.00 , con una desviación estándar de 21.20 . El puntaje máximo obtenido fue de 108, y el mínimo de 1.

Se pretendía analizar también las diferencias que pudiera haber entre los términos incluidos en la prueba en razón de su registro y frecuencia. Es decir, se esperaba que las palabras más frecuentes fueran más conocidas que aquellas de poca frecuencia y pertenecientes al registro oral. Los resultados que se obtuvieron corroboran esta hipótesis, ya que los términos que aparecen con más frecuencia en el corpus, también resultaron ser aquellos que los entrevistados conocieron más, mientras que los términos que aparecen en el corpus con menos frecuencia obtuvieron puntajes más bajos.

Por otra parte, también se pudo percibir la diferencia entre el registro oral y el escrito, ya que los términos que solamente aparecieron en entrevistas con informantes resultaron los más complicados para los entrevistados. En la Gráfica (1) se pueden ver estas diferencias. Es importante aclarar que dado que las categorías no contienen el mismo número de palabras, se normalizaron los resultados utilizando porcentajes. Los entrevistados conocieron el $65.3 \%$ de las palabras de alta frecuencia, el $53.5 \%$ de las palabras de baja frecuencia y el 33.9\% de las palabras de registro oral.

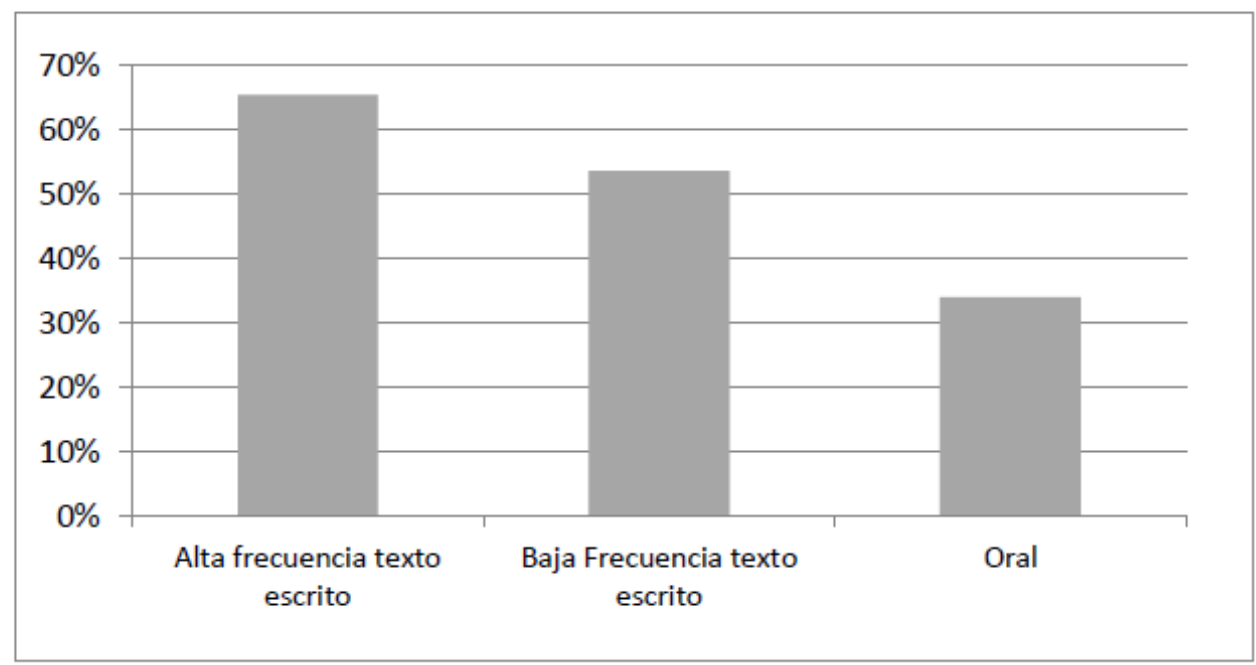

Gráfica 1. Porcentaje de palabras conocidas según su frecuencia. 


\subsection{Edad}

Una de las hipótesis que se tenía en relación al narcolenguaje, era que, al percibirse como un fenómeno relativamente nuevo, o por lo menos que en años recientes es que se ha constituido en el fenómeno social de mayor relevancia en México - tanto que algunas de sus expresiones culturales como la moda y la música se han vuelto muy populareslas generaciones más jóvenes conocerían más el narcolenguaje.

No obstante, al correlacionar el puntaje de conocimiento de narcolenguaje con la variable "edad" se encontró que estadísticamente no existe una correlación; es decir, la edad no parece ser un factor determinante en el conocimiento del narcolenguaje. Esto en el análisis de la puntuación global.

Al realizar el análisis de cada una de las tres categorías de palabras que se consideraron, se encontró una correlación positiva y moderadamente significativa en las palabras de registro escrito de alta frecuencia; $(r=.266, n=83 ; p=.015)$. Es decir, a mayor edad se conocen más las palabras que son muy frecuentes en textos escritos. Dado que las palabras que se ubicaron en esta categoría provenían mayormente de periódicos, revistas y libros especializados en el tema del narco, es posible conjeturar que este tipo de textos son más leídos por las personas adultas que por los jóvenes; aunque esto no se pudo demostrar estadísticamente, por lo que esta hipótesis se rechazó.

En cuanto a las otras categorías (baja frecuencia en textos y palabras de registro oral) no se encontró que hubiera alguna relación entre la edad y el conocimiento de este tipo de palabras.

\subsection{Género}

Se ha encontrado que los hombres tienen la tendencia a usar un lenguaje más rudo, o más "viril", que el que utilizan las mujeres; esto por lo menos en las clases medias-bajas. Conde Silvestre (2007) habla acerca de cierta reticencia de los varones a adoptar los cambios iniciados por hablantes femeninos de su misma generación. Debido a la propia naturaleza marginal del mismo, suponíamos que en este caso serían los hombres quienes tendrían un mayor conocimiento del léxico usado por el narco. 
Al hacer una comparación de medias (Prueba T), se encontró que la variable "género" sí está relacionada con el conocimiento del narcolenguaje en favor de los hombres; $(t=-5.22$, g.l.=81, p=.000). En puntaje global, como se indica en la Gráfica (2), las mujeres solamente el $40 \%$ de la máxima puntuación posible mientras que los hombres obtuvieron el $58 \%$.

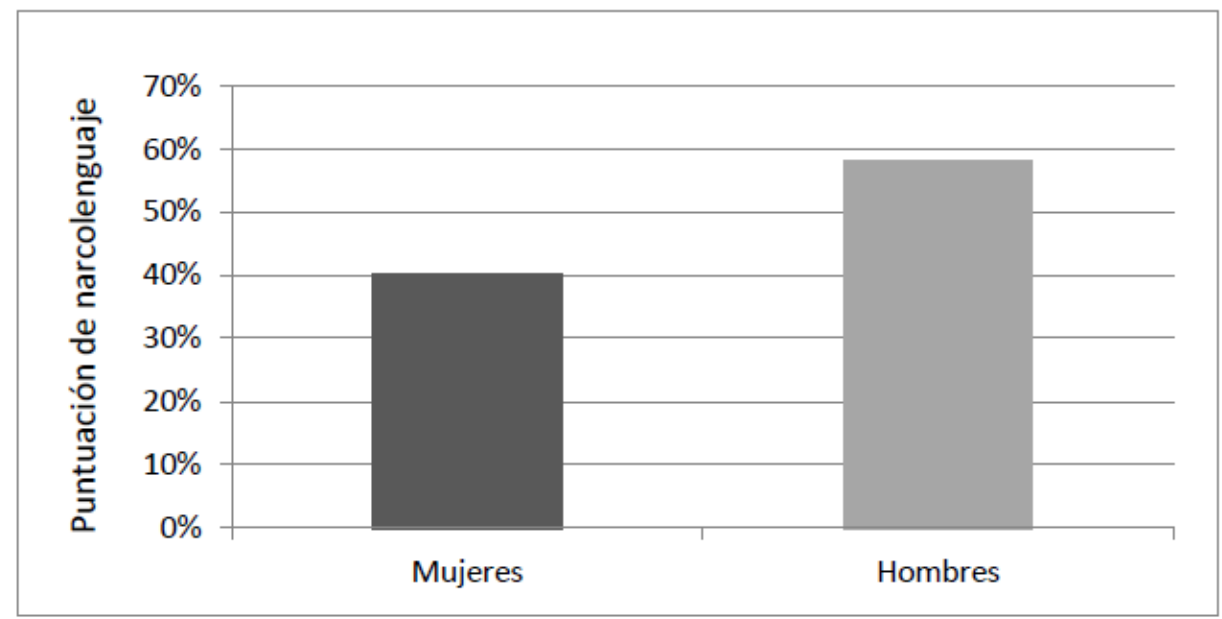

Gráfica 2. Puntuación de narcolenguaje en relación al género.

La tendencia a analizar las palabras en conjunto se mantuvo al analizar cada categoría por separado, obteniendo los hombres una media de conocimiento de narcolenguaje mayor que el de las mujeres en todas las categorías (Gráfica (3)).

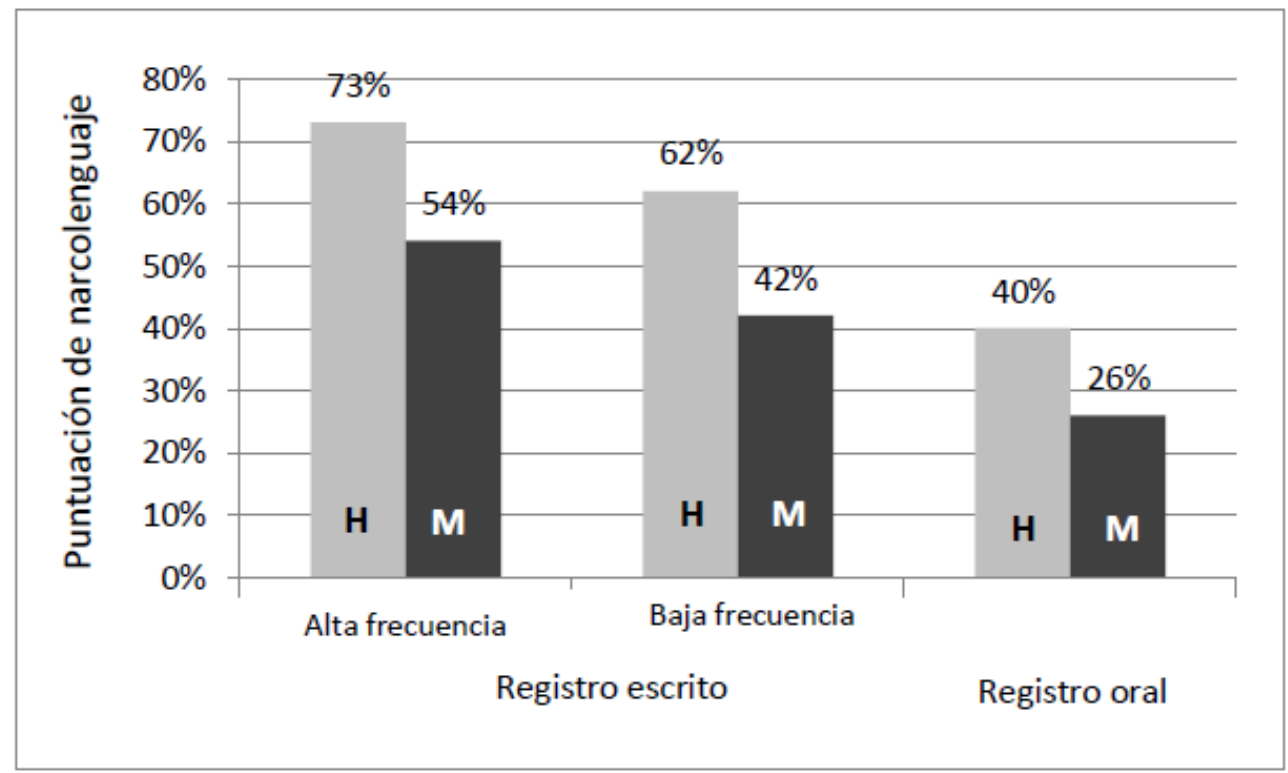

Gráfica 3. Conocimiento de narcolenguaje por frecuencia y por género. 


\subsection{Clase social}

Romaine (2009) afirma que, en general, el uso de formas no estandarizadas se incrementa entre más informal sea el estilo y más bajo el estatus social de las personas. Se esperaba que fueran los miembros de la clase social baja quienes tuvieran un mayor conocimiento del lenguaje narco. Para revisar esto, se corrieron pruebas de correlación tanto de todos los términos en conjunto como de cada una de las categorías de frecuencia por separado.

Los resultados mostraron que, estadísticamente, no hay evidencia suficiente para hacer una aseveración categórica en el sentido de que las personas de clase baja conocen más narcolenguaje, únicamente hay una correlación negativa moderadamente significativa entre clase social y el conocimiento de palabras de registro oral; $(r=.247$, $\mathrm{n}=83 ; \mathrm{p}=.024)$. De este resultado podemos reflexionar que, dado que las palabras pertenecientes a esta categoría son aquellas utilizadas por las personas que se dedican al comercio u consumo de drogas, es en las clases bajas donde habría un mayor conocimiento de estas palabras; ya que la drogadicción y el trasiego de drogas es mucho más evidente en las zonas marginales de las ciudades.

\subsection{Cercanía con el fenómeno narco}

Conde Silvestre (2007) afirma que la interacción cotidiana con los demás promueve el proceso de cambio, de modo que aquí se pretendía conocer si la presencia palpable del fenómeno narco en la vida cotidiana, entendiendo por esto el contacto con personas dedicadas al tráfico y al consumo de las drogas, influye en la cantidad de narcolenguaje que las personas incorporan en su habla cotidiana.

Se llevó a cabo una prueba Anova de un factor y se encontraron los siguientes resultados: $\mathrm{F}=(3,82)=8.1, \mathrm{p}=.000$. Esto indica que las variables están asociadas, por tanto a mayor cercanía de las personas con la narcocultura se conoce más narcolenguaje. De las personas entrevistadas, 14 mencionaron no tener ningún contacto con el comercio y consumo de drogas, 21 dijeron tener poco contacto, 25 dijeron tener un contacto moderado y 23 un contacto alto. Estos datos por si mismos son importantes en tanto 
muestran que la presencia del narco es patente en la región y solamente el $16 \%$ de los entrevistados dice estar completamente aislado del consumo y la distribución de drogas ilegales.

Al revisar las categorías de palabras de manera individual se encontró que para las tres categorías (alta frecuencia, baja frecuencia y registro oral) existe una asociación con los factores mencionados anteriormente: conocer a consumidores de droga, conocer a vendedores y vivir cerca de un lugar en el que se expende droga. En la Gráfica (4) podemos ver esta variable contrastada con el conocimiento global del narcolenguaje.

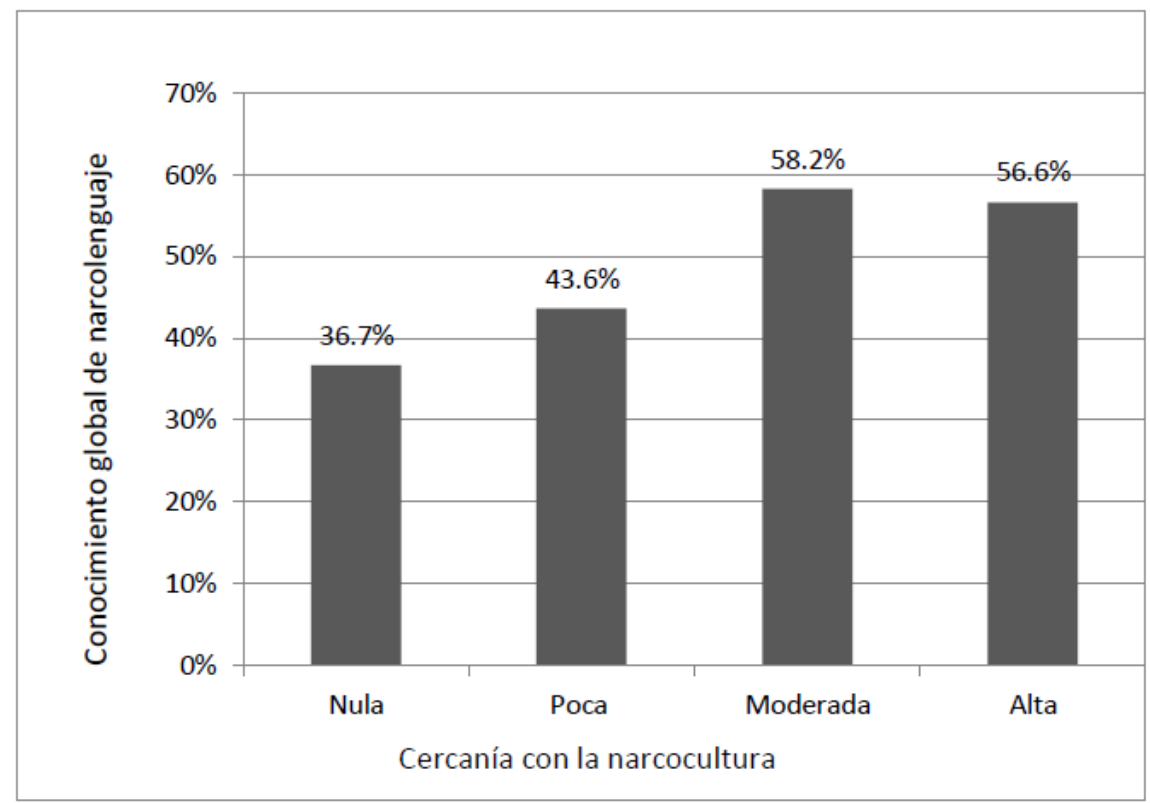

Gráfica 4. Puntuación de conocimiento global de narcolenguaje y cercanía con la narcocultura.

\subsection{Consumo de narcocultura}

La cultura del narco se manifiesta en la sociedad de diversas formas, de manera directa, como se explicaba en el punto anterior, o a través de los medios de comunicación. Probablemente la prensa ha sido el medio que más ha contribuido al imaginario social que se ha creado del fenómeno narco. Este fenómeno también se hace presente en manifestaciones artísticas como la música, el cine y la televisión. La hipótesis que consideramos a este respecto implicaba que quienes se interesaban por leer acerca 
del narco, participar en foros de internet especializados, y consumir las manifestaciones artísticas de la narcocultura, también habrían de conocer una narcolenguaje más amplio.

Para comprobar esta hipótesis se corrió una prueba de correlación entre el puntaje de "hábitos de consumo cultural" y la puntuación general de conocimiento narco, y por categorías de frecuencia. En la prueba de correlación de manera global se encontraron que las variables están correlacionadas positivamente: $r=.480, p=.000$. ; $n=83$ (Gráfica (5)).

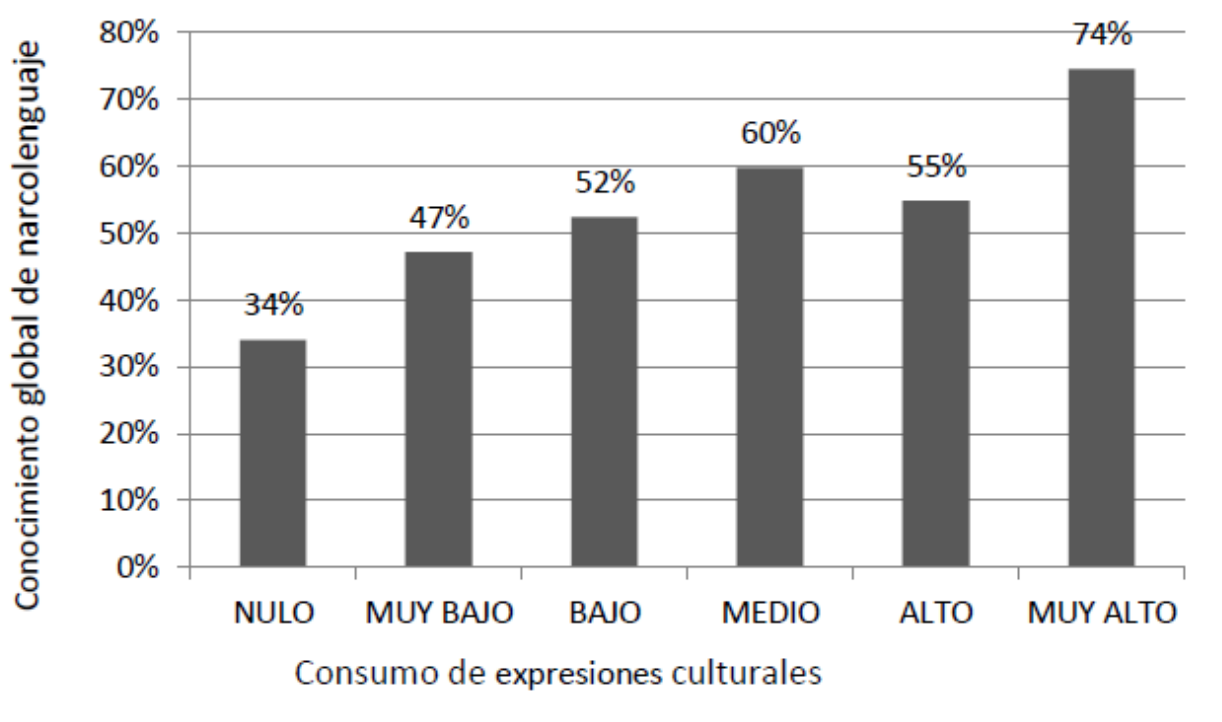

Gráfica 5. Puntaje de conocimiento global de narcolenguaje y consumo de expresiones culturales.

Como se puede ver en la tabla anterior, las personas que se ubicaron en los tres grados de mayor consumo de manifestaciones de la narcocultura demostraron tener un conocimiento del léxico narco más elevado que las personas que manifestaron poco interés por las representaciones de la narcocultura en los medios y en expresiones artísticas.

Es interesante notar que el $68 \%$ de las personas encuestadas se ubican en un nivel de consumo de narcocultura de bajo a nulo; nulo=12, muy bajo=29, bajo=16. El restante $32 \%$ se ubicó en: promedio=17 personas, alto=6, y muy alto= 3 . Lo cual indica que este tipo de expresiones siguen manteniendo cierto grado de la marginalidad. 
Para saber cuál de estas representaciones culturales aporta más al conocimiento del narcolenguaje, se realizaron pruebas T para cada uno de los cinco rubros (Gráfica 6).

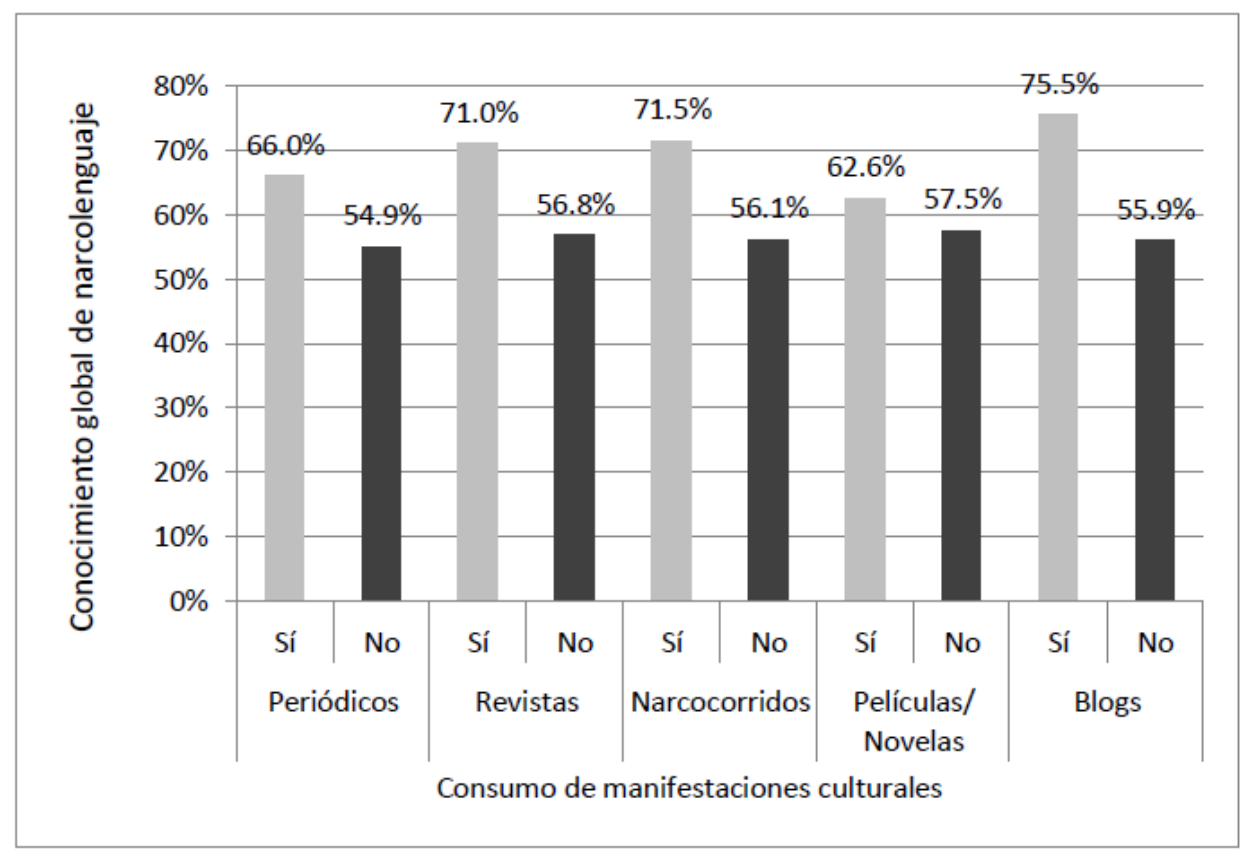

Gráfica 6. Conocimiento global de narcolenguaje y consumo de manifestaciones culturales por separado.

En la gráfica se puede ver que la única manifestación cultural que parece no contribuir mucho son las películas o telenovelas acerca del narco, que ahora se han vuelto una moda. Los demás rubros sí muestran ser significativos de manera individual. Leer el periódico $(t=2.450, p=.016)$, leer revistas de política $(t=2.776, p=.007)$, escuchar narcocorridos $(t=3.105, p=.003)$, ver películas o telenovelas $(t=1.079, p=.284)$, frecuentar blogs ( $t=3.834, p=.000)$; en todos los casos $n=83$. Las dos manifestaciones culturales que parecen estar relacionadas con un alto conocimiento del narcolenguaje son también las de un uso más restringido, ya que solamente 12 personas afirmaron escuchar narcocorridos y 19 frecuentar sitios especializados en el tema del narco.

Al comparar los resultados de las tres categorías distintas se puede constatar la relación con los hábitos de consumo de información y expresiones culturales de las personas. Por ejemplo, las palabras consideradas de alta frecuencia en textos escritos, términos que típicamente utiliza la prensa para referirse al fenómeno narco, son poco conocidos por las personas que no acostumbran leer el periódico. No obstante, la mayoría de las palabras ubicadas en esta categoría son ampliamente difundidas, y en general se obtuvieron puntuaciones altas. Estadísticamente, los cuatro rubros que se 
relacionan más con el conocimiento del lenguaje de alta frecuencia fueron: Leer periódicos $(t=2.729, p=.008)$, leer revistas $(t=2.601, p=.011)$, escuchar narcocorridos $(t=2.431, p=.017)$, frecuentar blogs $(t=2.645, p=.010)$ (Gráfica 7$)$.

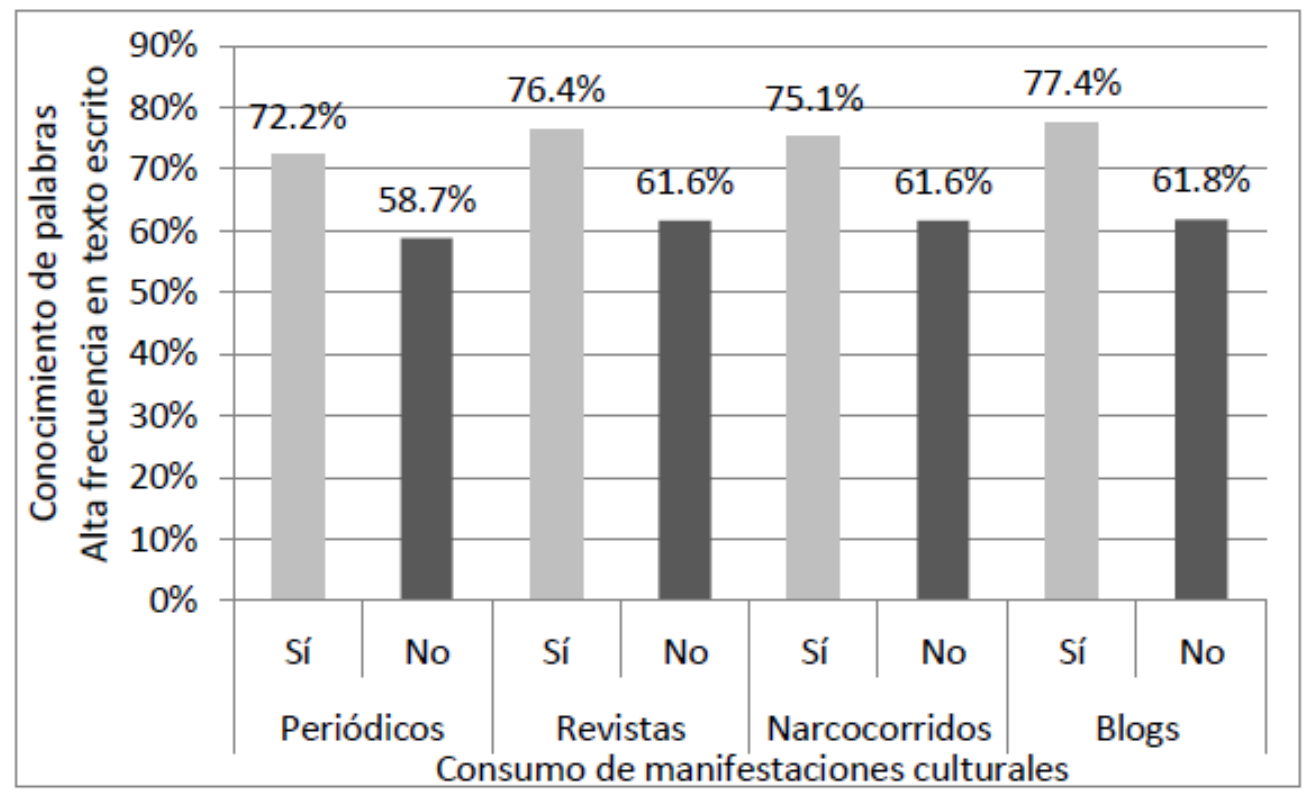

Gráfica 7. Conocimiento de palabras de alta frecuencia en texto escrito y consumo de manifestaciones culturales.

Las palabras que se categorizaron como de frecuencia baja en textos escritos, son de uso un poco más restringido en relación a la especialización de los textos (libros o revistas especializadas en el tema del narco), o incluso a un cierto carácter dialectal. Probablemente debido a ello es que las dos actividades de consumo cultural que resultaron con una asociación más fuerte con el conocimiento de estas palabras fueron la lectura de revistas especiadas y el acceso a sitios de internet especializados en el tema del narco.

Es interesante notar que las personas que afirman frecuentar sitios de internet especializados en el fenómeno narco, como blogs, fueron quienes normalmente obtuvieron puntuaciones más altas en todas las categorías de palabras y en forma global; y esto nos hace suponer que son personas muy interesadas en el fenómeno del narco y en sus diferentes manifestaciones. 
Estadísticamente los únicos dos rubros que manifestaron una relación significativa con el conocimiento de los términos de frecuencia baja en texto escrito fueron: leer revistas de política y temas del narco $(t=2.547, p=.013)$, y frecuentar blogs $(t=2.645$, $\mathrm{p}=.010)$ (Gráfica 8).

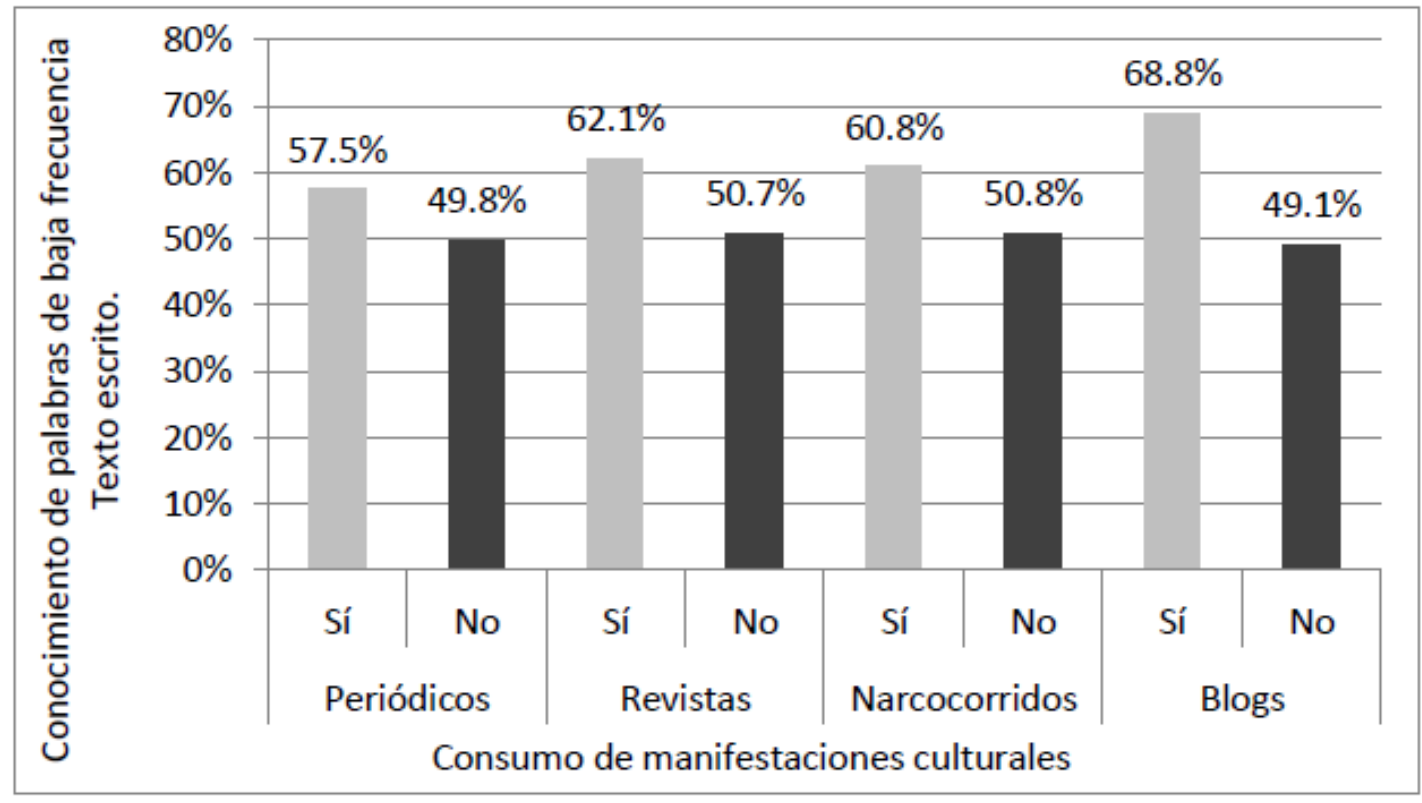

Gráfica 8. Conocimiento de palabras de frecuencia baja en texto escrito y consumo de manifestaciones culturales.

En la categoría de palabras de registro oral se encontró una vez más que los usuarios de blogs demuestran un alto conocimiento de éstas; sin embargo, obtienen un puntaje más alto aquellas personas que afirman escuchar narcocorridos.

Antes se hablaba acerca de las características de los narcocorridos como generadores y modeladores del lenguaje utilizado por las personas que viven del tráfico de sustancias ilegales. Esta prueba parece demostrar dicha idea, ya que esta actividad fue la que tuvo una asociación más fuerte con el conocimiento de palabras usadas por informantes y usuarios de blogs; las cuales mostraron ser las menos conocidas por las personas de manera general.

Los datos más relevantes que se obtuvieron en la prueba estadística fueron los siguientes. Leer periódicos $(t=2.072, p=.041)$, leer revistas de políticas $(t=2.417, .018)$, escuchar narcocorridos ( $t=4.033, p=.000)$, y frecuentar blogs ( $t=3.177, p=.002)$ (Gráfica 9). 


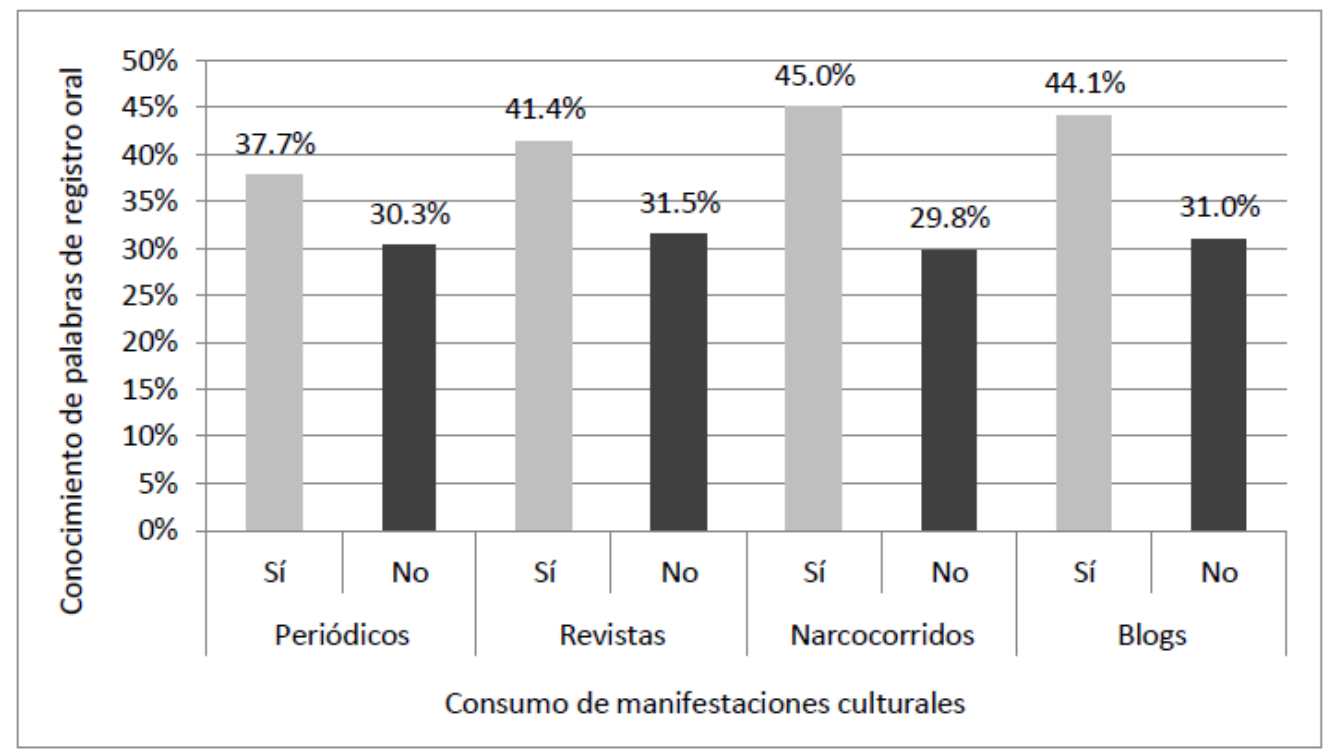

Gráfica 9. Conocimiento de palabras de registro oral y consumo de manifestaciones culturales.

\section{Conclusiones}

En esta investigación se cumplieron varios de los objetivos señalados antes, primeramente se identificó el léxico utilizado para referirse al fenómeno narco, ya sea por miembros de las comunidades relacionadas con el tráfico de drogas, por la prensa, o por la población en general. De esta terminología se realizó un estudio lexicológico, a través de un análisis de los patrones de comportamiento y asociación de estos términos.

De este análisis lexicológico y lexicográfico realizado con programas de cómputo actuales se pudo llegar a una conclusión importante; el narcolenguaje no puede tratarse como un ente homogéneo; existen diferentes sub-lenguajes al interior del narcolenguaje, los cuales son utilizados en diferentes espacios sociales.

Tomando como base este trabajo terminológico, se revisaron las dinámicas de diseminación de estos términos en diferentes estratos de la sociedad de Baja California y se analizaron las incidencias de diferentes variables sociales en el conocimiento de este tipo de lenguaje. Se pudo concluir por ejemplo, que, a pesar de la gran atracción mediática que ha generado el lenguaje del narco, el lenguaje utilizado por la prensa es conocido principalmente por las clases sociales que se interesan por mantenerse 
informadas. El lenguaje más marginal, utilizado por los traficantes de drogas y consumidores, es más conocido por los integrantes de las clases bajas.

Otros hallazgos interesantes son: que los hombres conocen más narcolenguaje que las mujeres, que las personas que conocen más narcolenguaje tienen entre 25 y 40 años. Se encontró que la cercanía del fenómeno narco está relacionada con el conocimiento del narcolenguaje, y que muchas personas perciben esta cercanía en su cotidianidad, lo cual tal vez explique porque a pesar de que las personas no escuchen narcocorridos o visiten sitios de internet en realidad conocen mucho de este lenguaje.

Finalmente queremos concluir diciendo que esta investigación es descriptiva, pero ha sido un estudio sistemático que hace una contribución al campo de la sociolingüística, especialmente a los estudios de variación léxica y a la lexicografía, ya que el enfoque multidisciplinar que se ha seguido es innovador. Consideramos que supone una base para posteriormente profundizar aún más en el conocimiento del narcolenguaje al enfocarse más en alguno de los sub-géneros, revisar patrones morfológicos, realizar estudios discursivos, etc. Además podría ser de utilidad para referencia a futuros estudios sociolingüísticos que tengan que ver con variación léxica o con dialectos sociales de distintos tipos en Baja California.

\section{Referencias}

BAKER, P. (2010) Sociolinguistics and corpus linguistics, Edinburgh: Edinburgh University Press.

BLAKE, B. (2010) Secret language. Codes, tricks, spies, thieves, and symbols, New York: Oxford University Press.

CONDE SILVESTRE, J. C. (2007) Sociolingüística histórica, Madrid: Gredos.

GeeraerTs, D. (2008) "Prototypes, stereotypes, and semantic norms", en G. Kristiansen \& R. Dirven, Cognitive Sociolinguistics. Language variation, cultural models, social systems, Berlin: Mouton de Gruyter, 21-44.

LaRa Ramos, L. F. (2006) Curso de lexicología, México: El Colegio de México.

ROLDÁN QUIÑOÑEZ, L. F. (2006) Diccionario irreverente de política mexicana, México: Grijalbo.

ROMAINE, S. (2009) "Corpus linguistics and sociolinguistics", en A. Lüdeling, \& M. Kytö, Corpus linguistics. An international handbook, vol 1, Berlin: Walter de Gruyter, 96-111. 